\title{
Tigecycline induced acute pancreatitis: Suspicion is the first step in diagnosis
}

\author{
(D)Alpaslan Tanoğlu ${ }^{1}$, ĐEylem Çağıltay ${ }^{2}$ \\ ${ }^{1}$ Health Sciences University, Sancaktepe Sehit Prof. Dr İlhan Varank Training and Research Hospital, Department of Internal Medicine and \\ Gastroenterology, İstanbul, Turkey \\ ${ }^{2}$ Health Sciences University, Sultan Abdulhamid Han Training and Research Hospital, Department of Endocrinology and Metabolism, \\ İstanbul, Turkey
}

Cite this article as: Tanoğlu A, Çağıltay E. Tigecycline induced acute pancreatitis: Suspicion is the first step in diagnosis. Anatolian Curr Med J 2021; 3(3); 262-263.

\section{Dear Editor,}

Acute pancreatitis is an inflammatoryevent of the pancreas which may affect seriously all other tissues and organ systems (1-3). Generally, acute pancreatitis is induced by alcoholism or gallstones. However pathogenetic mechanisms of drug induced pancreatitis are not clearly understood (4). A minocycline derivative, tigecycline is a glycylcycline class of antibiotic which is structurally similar to the tetracyclines. It has perfect tissue and organ distribution and favorable microbial coverage, including many kind of Gram-positive and Gram-negative bacteria and also anaerob agents $(5,6)$. Tigecycline's side-effect profile is similar to the tetracyclines (7). Pancreatitis is a well-known adverse effect of tetracyclines (8). However, tigecycline-induced pancreatitis is an uncommon sideeffect. We report here a rare case of tigecycline-induced pancreatitis during the treatment of a diabetic food infection.

A 64-year-old man was admitted to hospital because of diabetic food infection requiring glisemic control and antibiotheraphy. He had a history of cholecystectomy operation nearly 5 years ago. His medical history consisted of no alcohol consumption for five years. At the beginning of his diabetic food treatment, teicoplanin 400 $\mathrm{mg}$ one per day and imipenem $500 \mathrm{mg}$ four times per day intravenous (iv) injection began empirically. Repetitive diabetic food wound culture was taken and they revealed acinotobacter spp. enfection that was sensitive to colistin, ceftasidime and doxycycline. The antibioteraphy was switched to tigecycline $100 \mathrm{mg}$ iv infusion, then $50 \mathrm{mg}$ iv twice a day and colistin iv $150 \mathrm{mg}$ twice a day. 9 days after the beginning of this therapy, gastro-intestinal symptoms, such as severe epigastric abdominal pain, loss of appetite, nausea and vomiting, were started. Serum amylase (761 U/L, 17-115 normal range) and lipase (426 U/L, 7-60 normal range), aspartate aminotransferase (251 $\mathrm{U} / \mathrm{L}$, normal $<40)$, alanine aminotransferase $(263 \mathrm{U} / \mathrm{L}$, normal $<40)$ levels and leucocytes $\left(11.9 \times 10^{3} / \mathrm{mm}^{3}, 4.0-\right.$ 10.5 normal range) counts were increased. Abdominal ultrasonography revealed enlarged edematous pancreas and he was diagnosed as 'acute pancreatitis'. Oral nutrition was stopped, he was hydrated intravenously and fed with parenteral nutrition. Computed tomography imaging could not be performed because of chronic renal failure and elevated creatinine levels. Tigecycline-induced pancreatitis was suspected and the drug was ceased. Then cefoperazone sulbactam and colistin therapy continued. Abdominal pain and vomiting resolved allowing the patient to receive oral alimentation after few days. Amylase and lipase values were decreased to normal levels within a few days. After few days with successful medications, the patient was discharged in a safe condition.

Acute pancreatitis is an important life-threatening pancreatic disease $(1,3)$ Many kind of drugs or toxins may cause acute pancreatitis (4). In the way to exact diagnosis of drug-induced acute pancreatitis, the first step is being suspicious from the currently used drugs. Tigecycline is a globally used wide spectrum antibiotic which is used for many kinds of infectious circumstances (8). It has generally has a safe side effect profile and its side-effect range is similar to the tetracyclines (7). In this current case, we used Naranjo adverse drug reaction probability scale for estimating the probability of adverse effect and a score of 5 was reached. Thus we suggest that a probable relationship between tigecycline and acute pancreatitis is existing (9). 
Drug induced acute pancreatitis is an important and sometimes life threatening condition which should be diagnosed early and treated properly. Attention should be given to stop the treatment with tigecycline in patients with acute pancreatitis since it can cause life-threatening consequences.

Keywords: Tigecycline, acute pancreatitis, advers effect

\section{ETHICAL DECLARATIONS}

Informed Consent: Written informed consent was obtained from all participants who participated in this study.

Referee Evaluation Process: Externally peer-reviewed.

Conflict of Interest Statement: The authors have no conflicts of interest to declare.

Financial Disclosure: The authors declared that this study has received no financial support.

Author Contributions: All of the authors declare that they have all participated in the design, execution, and analysis of the paper, and that they have approved the final version.

\section{REFERENCES}

1. Tanoglu A, Yazgan Y, Kaplan M, et al. Trimetazidine significantly reduces cerulein-induced pancreatic apoptosis. Clin Res Hepatol Gastroenterol 2015; 39: 145-50.

2. Hançerli Y, Kaplan M, Tanoğlu A, et al. Efficacy of tocilizumab treatment in cerulein-induced experimental acute pancreatitis model in rats. Turk J Gastroenterol 2017; 28: 485-91.

3. Kaplan M, Yazgan Y, Tanoglu A, et al. Effectiveness of interleukin-1 receptor antagonist (Anakinra) on ceruleininduced experimental acute pancreatitis in rats. Scand J Gastroenterol 2014; 49: 1124-30.

4. Tanoglu A, Cagiltay E, Beyazit Y. Spiramycin-associated Acute Pancreatitis: Cause or Coincidence? Med Sci 2015; 4: 2352-5.

5. Giamarellou H, Poulakou Pharmacokinetic and pharmacodynamic evaluation of tigecycline. Expert Opin Drug Metab Toxicol 2011; 7: 1459-70.

6. Barbour A, Schmidt S, Ma B, Schiefelbein L, et al. Clinical pharmacokinetics and pharmacodynamics of tigecycline. Clin Pharmacokinet 2009; 48: 575-84.

7. Brink AJ, Bizos D, Boffard KD, et al. Guideline summary: appropriate use of tigecycline. S Afr J Surg 2012; 50: 20-1.

8. Lipshitz J, Kruh J, Cheung P, Cassagnol M. Tigecycline-induced pancreatitis. J Clin Gastroenterol 2009; 43: 93.

9. Naranjo CA, Busto U, Sellers EM, et al. A method for estimating the probability of adverse drug reactions. Clin Pharmacol Ther 1981; 30: 239-45. 